Research Paper

\title{
Development of RFLP-PCR method for the identification of medically important Aspergillus species using single restriction enzyme $M w o I$
}

\author{
K. Diba ${ }^{1}$, H. Mirhendi ${ }^{2}$, P. Kordbacheh ${ }^{3}$, S. Rezaie ${ }^{4}$ \\ ${ }^{1}$ Cellular and Molecular Research Center, School of Medicine, Urmia University of Medical Sciences, \\ Urmia, West Azarbayejan, Iran. \\ ${ }^{2}$ Department of Parasitology and Mycology, School of Public Health and Institute of Public Health \\ Research, Tehran University of Medical Sciences, Tehran, Iran. \\ ${ }^{3}$ Department of Parasitology and Mycology, School of Public Health and Institute of Public Health \\ Research, Tehran University of medical sciences, Tehran, Iran. \\ ${ }^{4}$ Department of Parasitology and Mycology, School of Public Health and Institute of Public Health \\ Research, Tehran University of medical sciences, Tehran, Iran.
}

Submitted: September 18, 2012; Approved: March 14, 2014.

\begin{abstract}
In this study we attempted to modify the PCR-RFLP method using restriction enzyme MwoI for the identification of medically important Aspergillus species. Our subjects included nine standard Aspergillus species and 205 Aspergillus isolates of approved hospital acquired infections and hospital indoor sources. First of all, Aspergillus isolates were identified in the level of species by using morphologic method. A twenty four hours culture was performed for each isolates to harvest Aspergillus mycelia and then genomic DNA was extracted using Phenol-Chloroform method. PCR-RFLP using single restriction enzyme $M w o I$ was performed in ITS regions of rDNA gene. The electrophoresis data were analyzed and compared with those of morphologic identifications. Total of 205 Aspergillus isolates included $153(75 \%)$ environmental and $52(25 \%)$ clinical isolates. A. flavus was the most frequently isolate in our study (55\%), followed by A. niger $65(31.7 \%)$, A. fumigatus 18(8.7\%), A. nidulans and A. parasiticus 2(1\% each). MwoI enabled us to discriminate eight medically important Aspergillus species including A. fumigatus, A. niger, A. flavus as the most common isolated species. PCR-RFLP method using the restriction enzyme $M w o I$ is a rapid and reliable test for identification of at least the most medically important Aspergillus species.
\end{abstract}

Key words: Aspergillus, identification, MwoI.

\section{Introduction}

Invasive filamentous fungal infections have emerged as important human diseases in recent years, particularly in the patients who receiving aggressive chemotherapy regimens and immunosuppressive agents (Walsh and Francesconi, 1995). Aspergillus species are most abundant and widely distributed in soil, water, air, seeds and foods (Anaissie and McGinnis, 2003). These molds are associated with allergic bronchopulmonary disease, mycotic keratitis, otomycosis, nasal sinusitis and invasive infection. Aspergillus fumigatus remains the most frequent cause of invasive aspergillosis (IA), although at least 30 other species, primarily $A$. flavus, $A$. terreus, $A$. niger, A. nidulans, $A$. ustus and $A$. versicolor have been associated with human disease (De Aguirre et al., 2004). In some institutions, $A$. terreus is becoming more common and is of concern because it is less susceptible to antifungal drugs such as amphotericine-B than A. fumigatus. Similarly Aspergillus nidulans has also been reported as less susceptible to amphotericine-B than $A$. fumigatus and although it rarely causes invasive disease, A.ustus is less susceptible than $A$. fumigatus to antifungal medications such as voriconazole (Zhao and Kong, 2001). Therefore, accurate and timely

Send correspondence to K. Diba. Cellular and Molecular Research Center, School of Medicine, Urmia University of Medical Sciences, Urmia, Iran. E-mail:kambiz37diba@gmail.com. 
species identification is important to manage IA as well as for surveillance and epidemiological purposes.

Identification of Aspergillus species has traditionally relied on macroscopic colony characteristics and microscopic morphology (Diba et al., 2007). Recently, comparatively simpler and more efficient molecular techniques have been adapted to detect and identify pathogenic fungi including Aspergillus species. In particular, because of its speed, reproducibility, high sensitivity and specificity, Polymerase Chain Reaction (PCR)-based tests have increasingly been used in laboratories to identify numerous fungal species (Canbe et al., 2002). Several methods have been reported for DNA-based identification of Aspergillus spp. including PCR-sequencing of the ITS region (Moody and Tyler, 1990) repetitive-sequence based PCR (Healy and Kontoianis, 2004), PCR-Enzyme Immune Assay (EIA) (De Aguirre and Choi, 2004), nested PCR targeting rDNA ITS regions (Zhao and Kong, 2001) and PCR-restriction fragment length polymorphism (PCR-RFLP) for identification of Aspergillus at the generic level (Mirhendi and Diba, 2007), as well as direct PCR/sequence (d-PCRs) (Moody and Tyler, 1990). In a study, we also introduced the molecular, PCR based method of single strand conformational polymorphism (SSCP) for the identification of medically important Aspergillus species (Diba and Mirhendi, 2008). In this study we attempted to modify the PCRRFLP method by using restriction enzyme $M w o$ I for the identification of most common hospital isolated Aspergillus species .

\section{Materials and Methods}

\section{Microorganisms}

Referenced cultures of Aspergillus species were obtained from the Japanese Collection of Microorganisms (JCM) including: A. flavus (JCM 2061), A. fumigatus (JCM 10253), A. niger (JCM 10254), A. nidulans (JCM 10227) and A. tereus (JCM 02728). Test samples were collected from clinical specimens including; sputum, sinus discharge, broncho alveolar lavage, nail scrapings; the environmental samples were included aerosols precipitated on Sabouraud medium (Difco) and surface swabs of indoor and outdoor environments, in a University hospital, Urmia, Iran.

\section{Culture preparation and morphological study}

Morphologic identification of all standard and hospital isolated Aspergillus spp. was performed by using four enrichment media: Czapek dox agar, Czapek yeast extract agar, Czapek yeast 20\% sucrose agar and malt extract agar (Diba and Mirhendi, 2007). For the molecular study, Aspergillus mycelia were harvested from sabouraud glucose broth (Difco) cultures after $24-48 \mathrm{~h}$ at $30^{\circ} \mathrm{C}$ on a shaking platform. The mycelia were recovered on a $0.45 \mu \mathrm{m}$ pore size filter and washed with TRIS- EDTA buffer and sterile deionised water. Aliquots of the fungal hypha were stored frozen at $-20{ }^{\circ} \mathrm{C}$ until use.

\section{DNA extraction}

Aspergillus freeze mycelia were ground using a Micro-Multimixer (IEDA Trading Corporation, Japan) or shaking with same volume of 0.3 to $0.4 \mathrm{~mm}$ (d) glass beads. DNA isolation of Aspergillus cells was performed using phenol-chloroform method (Mirhendi et al., 2007; Diba et al., 2008).

\section{PCR amplification}

PCR was performed using $5 \mu \mathrm{L}$ of the DNA template to the PCR master mix including PCR buffer (20 mM Tris$\mathrm{HCL}$ at $\mathrm{pH} 8.0), 50 \mathrm{mM} \mathrm{MgCl} 2,0.1 \mathrm{mM}$ each of forward (ITS: 5-TCC GTA GGT GAA CCT GCG G-3) and reverse (ITS-4 5-TCC TCC GCT TAT TGA TAT GC-3) primers (the universal primers synthesized by the UNMC, Eppley molecular biology lab), and 1.5 U of Taq DNA polymerase. Thermal protocol was use as our last studies on Aspergilli $(8,6)$. After amplification the products were stored at $4{ }^{\circ} \mathrm{C}$ until used.

\section{Restriction Enzyme Digestion}

Studying the literature and using molecular analyzer software (DNASIS Japan), we selected $M w o I$ which was a desirable restriction enzyme with differential cutting patterns for the identification of medically important Aspergillus species. For restriction digestion, $13 \mu \mathrm{L}$ of each PCR product was directly digested with by $5 \mathrm{U}(0.5 \mu \mathrm{L})$ of the restriction enzyme $M w o \mathrm{I}, 1.5 \mu \mathrm{L}$ of the related buffer, and incubated at $37^{\circ} \mathrm{C}$ for $180 \mathrm{~min}$ (Diba and Mirhendi, 2008). Digested PCR products were subjected to electrophoresis in a $2 \%$ agarose gel and visualized by gel doc UV system.

\section{Results}

Studying a lot of well known restriction enzymes with various digestion patterns, we chose $\mathrm{MwoI}$, as appropriate restriction enzyme with a differential power for nine of the 18 most medically important Aspergillus species tested in the present study. Amplification of rDNA in ITS1-5.8-ITS2 region for standard strains of Aspergillus species including A. fumigatus, A. flavus, A. niger, A. nidulans and $A$. terreus resulted PCR bands ranging 570-598 bp. Against PCR produced bands, digestion with MwoI in RFLP resulted different size bands of agarose gel electrophoresis (Figure 1). The MwoI cutting patterns for each Aspergillus species was completely specific, so that none of the eight Aspergillus species as mentioned Table 1 was mistaken from another. The method was tested for more 52 Aspergillus clinical isolates of nail scraping and sinus discharge specimens, sputum, biopsy materials and ear 


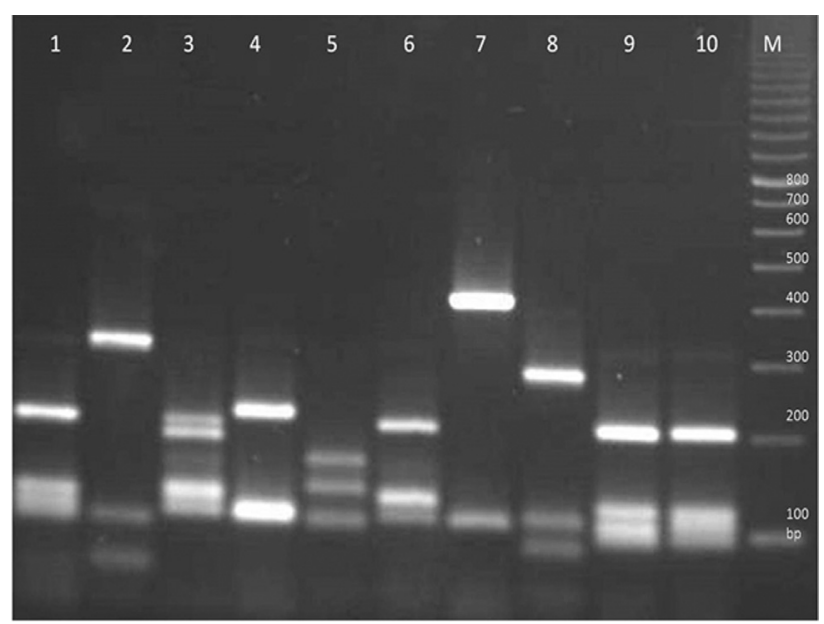

Figure 1 - Agarose gel electrophoresis of ITS1-ITS2 PCR products of standard Aspergillus species after digestion with the restriction enzyme MwoI Lanes 1-9: A. fumigatus, A. flavus, A. niger, A. terreus, A. nidulans, A. clavatus, A. ochraceus, A. amstelodami and A. fischeri. Lane 10: N. quadricincta (Digestion pattern is similar to A. fumigatus). Lane M $100 \mathrm{bp}$ ladder.

swabs, as well as 153 environmental specimens including indoor surfaces swabs.

During 18 month of our study, totally 205 isolates including 11 Aspergillus species were identified (Table 2). Aspergillus isolates included 153 (75\%) environmental and $52(25 \%)$ clinical isolates. Among all, A. flavus was the most common species $(55.0 \%)$ isolated from both clinical specimens followed by $A$. niger (31.7\%), A. fumigatus (8.7\%), A. nidulans (1.0\%) and A. tereus (0.5\%). Among environmental Aspergillus isolates, also A. flavus was found as the most frequent species followed by A. niger, $A$. fumigatus and the other Aspergillus species listed in Table 2 .
Table 1 - The fragment size of ITS1-ITS2 PCR products after digestion with restriction enzyme $\mathrm{MwoI}$ for some medically important Aspergillus species.

\begin{tabular}{lc}
\hline Species & Cutting size \\
\hline A fumigatus & $207,125,108,29,21,9$ \\
A. flavus & $325,98,65,40,20$ \\
A. niger & $192,175,120,108,30,21,9$ \\
A. terreus & $220,109,106,96,29,9$ \\
A. nidulans & $162,135,104,31,29,9$ \\
A. clavatus & $210,125,106$ \\
A. ochraceus & $420,90,39,9$ \\
A. amstelodami & $286,106,100,29,9$ \\
N. ficheri & $200,140,120$ \\
\hline
\end{tabular}

*: Neosrtorya ficheri.

\section{Discussion}

Use of molecular methods is preferred for the identification of medically important Aspergillus species comparing morphological tests which have some disadvantages including, delayed production or lack of characteristic morphologic features on culture media, lack of the sexual reproduction cycle in some Aspergillus species which makes difficult the identifications and similarity of microscopic or macroscopic characteristics in the species level and being risky for the health of laboratory personnel (Pryce and Palladino, 2006). The rDNA contains several regions of highly conserved sequence useful for obtaining proper sequence alignments. Most previous publications on PCR-based detection or identification systems were relevant to the sequencing rDNA $18 \mathrm{~S}$ or $28 \mathrm{~S}$ regions as target

Table 2 - Frequency of Aspergillus species isolated from clinical and environmental sources.

\begin{tabular}{|c|c|c|c|c|c|c|}
\hline \multirow[t]{2}{*}{ Aspergillus species } & \multicolumn{2}{|c|}{ Clinical isolates } & \multicolumn{2}{|c|}{ Environmental isolates } & \multicolumn{2}{|c|}{ Total } \\
\hline & Number & $\%$ & Number & $\%$ & Number & $\%$ \\
\hline A.flavus & 36 & 69.2 & 76 & 49.7 & 112 & 55 \\
\hline A. niger & 4 & 7.6 & 61 & 39.8 & 65 & 31.7 \\
\hline A. fumigatus & 7 & 13.5 & 11 & 7.2 & 18 & 8.7 \\
\hline A. nidulans & 1 & 1.9 & 1 & 0.6 & 2 & 1.0 \\
\hline A. terreus & 1 & 1.9 & 0 & 0 & 1 & 0.5 \\
\hline A. parasiticus & 1 & 1.9 & 1 & 1.6 & 2 & 1.0 \\
\hline A. penicilloides & 0 & 0 & 1 & 0.6 & 1 & 0.1 \\
\hline A. tamarii & 0 & 0 & 1 & 0.6 & 1 & 0.5 \\
\hline A. ochraceus & 1 & 1.9 & 0 & 0 & 1 & 0.5 \\
\hline A. sojae & 1 & 1.9 & 0 & 0 & 1 & 0.5 \\
\hline A. niveus & 1 & 1.9 & 1 & 0.6 & 1 & 0.5 \\
\hline Total & 52 & 100 & 153 & 100 & 205 & 100 \\
\hline
\end{tabular}


DNA, however, the sequences in these regions looks conserved across a wide range of fungi. It is therefore difficult to design truly species-specific primers to make differentiations by PCR amplifications. Several studies have shown that ITS-2 length polymorphism and/or RFLP patterns obtained after treatment of the PCR product with restriction endonuclease might be useful for the identification of fungal pathogens (Moody and Tyler, 1990).

Determination of ribosomal DNA sequences in ITS regions for discriminating Aspergillus species is so expensive that makes difficult using it as a routine diagnostic method in hospital medical labs or reference laboratories. Therefore, it has been often restricted to use for research aims (Yuan and Chen, 1995). Random amplified polymorphic DNA (RAPD) has proved useful for sub-typing and discriminating between Aspergillus strains (Walsh and Francesconi, 1995) however, the results are usually too complicated to interpret and also it is not sufficiently reliable for identification of clinically isolated fungi. There is sufficient sequence variability in rDNA region that can serve as an identification target for Aspergillus isolates by PCR-RFLP and ITS1 - ITS2 sub regions have been revealed as markers for discrimination among the species if using species-specific restriction enzyme analysis. De Aguirre developed a PCR-Enzyme Immuno Assay method using ITS1 and ITS2 region oligonucleotid probes to detect seven important Aspergillus species, but the method requires DNA sequencing followed by analysis on an automated capillary DNA sequencer which is time consuming and relatively expensive (De Aguirre and Hurst, 2004). Identification of the pathogenic Aspergillus species by nested-PCR using a mixture of specific primer to the DNA topoisomerase II gene was studied by Canbe so that $A$. fumigatus, A. flavus, $A$. nidulans and $A$. terreus were identified using the primer mix $\left(P_{S} I \mathrm{~V}\right)$ by nested PCR as a rapid (Canbe and Kikuchi , 2002). Simple and available method using primer sets designed for amplification of conserved genes from filamentous ascomycetes to identify them to the generic level (Yuan and Chen, 1995). In the present study, amplification of the 570-598 bp fragment (ITS1-5.8 S-ITS2) of Aspergillus species rDNA gene followed by $M w o$ I restriction in RFLP analysis allowed us to identify the most medically important Aspergillus species. Few investigators have reported use of RFLP with single restriction enzyme to identify Aspergilli to the level of species. De Aguirre (De Aguirre and Hurst, 2004) used the restriction enzyme StyI to show an identical pattern for Aspergillus species in the diagnosis of Aspergillus isolates at the generic level. An analysis of PCR-RFLP was used to identify five species of filamentous ascomycetes (Yuan and Chen, 1995). Dentis used RFLP to identify five Aspergillus species (Dentis, 2003). In this study we succeeded to differentiate at least 9 medically important Aspergillus species. Using this method, all tested standard strains were identified successfully except $N$. fischeri and A. amsteloidami.
The similarity between electrophoretic band patterns of Neosartoria fischeri and A. amsteloidami was exception as shown in the figure. Our findings of PCR-RFLP analysis on the clinical and environmental isolates were comparable with those of morphologic examinations. We recommend this method using restriction enzyme $M w o I$ as simple and reliable to identify of Aspergillus species isolated from some sources in a short time (5-6 h after a $24 \mathrm{~h}$ growth) in a clinical laboratory.

\section{Acknowledgments}

The authors thank Professor K. Makimura (Institute of Medical Mycology, Teikyo University, Tokyo, Japan) for the standard Aspergillus species and thank N. Jalalizand (Molecular Biologylogy lab, Institute of Health Researches, Esfehan, Iran), Dr. A. Namaki (Arefian General Hospital, Urmia, Iran) for their assistance. We also thank Dr. J. Smith (University of Adelaide, Australia) for her partly English edition of this paper. We thank deputy of Researches and Technology, Urmia University of Medical Sciences, Urmia, Iran and also Institute of Public Health, Tehran University of Medical Sciences, Tehran, Iran, for supporting some parts of this project.

\section{References}

Anaissie EJ, McGinnis MR (2003) Clinical Mycology. Churchill Livingstone Press, USA.

Canbe T, Yamaki K, Kikuchi A (2002) Identification of pathogenic Aspergillus species by Nested PCR using a mixture of species primers to DNA topoisomerase $\Pi$ gene. J Microb Immunol 46:846-848.

De Aguirre L, Hurst SF, Choi JS (2004) Rapid differentiation of Aspergillus species from other medically important opportunistic molds and yeasts by PCR-Enzyme Immunoassay. J Clin Microbiol 42:3495-3504.

Dentis N (2003) PCR -RFLP detection identification of species identification of fungal pathogens in patients with Febrile Neutrogena J Clin Mirobiol Infect 9:1991-2002.

Diba K, Kordbacheh P, Mirhendi H (2007). Identification of Aspergillus species using morphological characteristics. Pak J Med Scien 23:867-872.

Diba K, Mirhendi SH, Kordbacheh P, Jalalizand N (2008) Single Strand Conformation Polymorphism analysis of PCR-amplified rDNA to differentiate medically important Aspergillus Species. Ir J Publ Health 37:52-59.

Healy M, Reece K, Walton D, Kontoianis DP (2004) Identification to the species level and differentiation between strains of Aspergillus clinical isolates by Automated Repititive-Sequence-Based PCR. J Clin Microbiol 42:4016-4024.

Mirhendi SH, Diba K, Kordbacheh P (2007) Identification of pathogenis Aspergillus pecies by a PCR-restriction enzyme method J Med Microbiol 56:1568-1570.

Moody SF, Tyler BM (1990) Use of nuclear DNA Restriction Fragment Length Polymorphism to analyze the diversity of the Aspergillus flavus group; A. flavus, A. parasiticus and $A$. nomius. J Appl Environ Microbiol 56(8): 2453-2461. 
Moody SF, Tyler BM (1990) Restriction Enzyme Analysis of mitochondrial DNA of the Aspergillus flavus group; A. flavus, A. parasiticus and A. nomius. J Appl Environ Microbiol 56:2441-2452.

Pryce TM, Palladino S, Price DM, Gardam DJ, Murray RJ (2006) Rapid identification of fungal pathogens in BacT/Alert, BacTec and BBL MGIT media using polymerase chain reaction and DNA sequencing of the internal transcribed spacer regions. J Diag Microbiol Infec 54:289-297.

Travis H, Iwen PC, Hinrichs SH (2000) Identification of Aspergillus species using Internal Transcribed Spacer Regions 1 and 2. J Clin Microbiol 38:1510-51.
Walsh Tm, Francesconi A, Kasai M (1995) PCR and single strand conformational polymorphism for recognition of medically important opportunistic fungi. J clin Microbial 2:3216-20.

Yuan GF, Liu CS, Chen CC (1995) Differentiation of Aspergillus parasiticus from Aspergillus sojae by random amplification of polymorphic DNA. J Appl Environ Microbiol 61:23842387.

Zhao J, Kong F, Li R, Wang D (2001) Identification of Aspergillus fumigatus and isolated species and related species by Nested PCR targeting ribosomal DNA Internal Transcribed Spacer Region. J Clin Microbiol 39:2281-2286.

All the content of the journal, except where otherwise noted, is licensed under a Creative Commons License CC BY-NC. 